\title{
Ergonomie am Arbeitsplatz
}

Über die richtige Sitzhaltung während der zahnärztlichen Arbeit wird immer wieder berichtet. Im Laufe der Jahre wurde die stehende Behandlerposition von der sitzenden abgelöst. Damit Zahnarzt und Assistent gut arbeiten können, wurden auch die Behandlungsstühle weiterentwickelt. Eine verspannte Körperhaltung führt nämlich zu Kopf- und Rückenschmerzen.

Eine ausgewogene Körperhaltung ist nach Pirv et al. (2014) symmetrisch, den Oberkörper maximal um 20 Grad nach vorn gebeugt. Der Kopf sollte ebenfalls nicht mehr als 20 Grad geneigt werden. Auch Empfehlungen für die Beugung von Unterschenkel, Oberschenkel und Becken sind vorhanden: Unter- und Oberschenkel sollten einen Winkel von 105 bis 110 Grad, Oberschenkel und Hüfte von 45 Grad bilden. Die Füße sollten gerade auf dem Boden stehen. Der Abstand vom Auge zum Operationsfeld sollte 35 bis 40 Zentimeter betragen, die Okklusalflächen der Zähne parallel zum Oberkörper des Zahnarztes positioniert sein.

Damit die Sauerstoffversorgung der Muskulatur gewährleistet ist, sind kleine Bewegungen des Oberkörpers erwünscht. Insbesondere bei der Arbeit mit der Lupenbrille sollte darauf geachtet werden.

Kann der Zahnarzt mit einer korrekten Arbeitsposition neben dem physischen Wohlbefinden auch die Produktivität steigern? Dies untersuchten Abareshi et al. (2015). In einer Studie verglichen sie 158 Arbeiter einer Fabrik bezüglich der Produk- tivität und Motivation, wobei die Testgruppe ein Ergonomietraining zur Reduktion muskuloskeletaler Risikofaktoren erhalten hatte, die andere Hälfte der Arbeiter als Kontrollgruppe hingegen nicht. Vor dem Training unterschieden sich die beiden Populationen bezüglich der Motivation nicht, nach absolviertem Training jedoch signifikant.

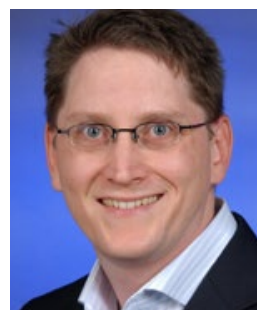

Prof. Dr. Dr. Felix P. Koch

Autor des Wissenschaftlichen Infodienstes, Lehrbeauftragter der Uniklinik Frankfurt, Praxisklinik für Mund- , Kiefer- und plastische Gesichtschirurgie in Wiesbaden. E-Mail: wid@fvdz.de

Der Wissenschaftliche Info-Dienst (WID) bringt alle zwei Monate Abstracts von wissenschaftlichen Publikationen und Vorträgen mit hohem Praxisbezug aus den Fachgebieten Parodontologie, Implantologie, Endodontie, Chirurgie, Prothetik, Zahnerhaltung bis zu Wissenswertem aus dem Internet. Für ein Jahresabo zahlen FVDZ-Mitglieder 33 Euro (Nichtmitglieder 49 Euro). Studentische FVDZ-Mitglieder können den WID für 15 Euro pro Jahr abonnieren. Informationen gibt es in der FVDZ-Bundesgeschäftsstelle bei Gabriele Brandenburg unter der Telefonnummer 0228 - 855762 oder unter www.fvdz.de.

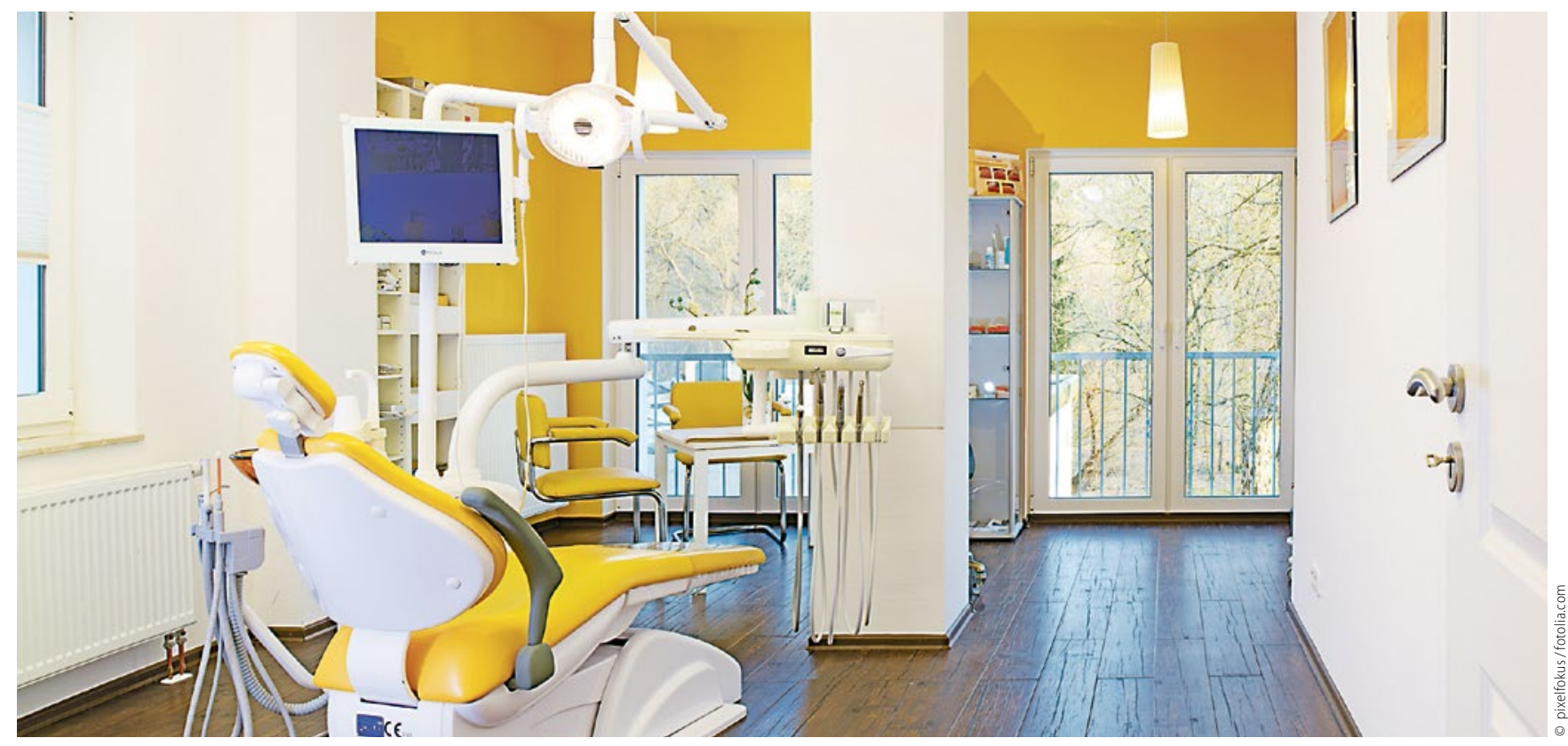

